\title{
THE COMPANION TO THE CENTRAL MIRA STAR OF THE PROTOPLANETARY NEBULA OH $231.8+4.2$
}

\author{
C. Sánchez Contreras, ${ }^{1}$ A. Gil de Paz, ${ }^{2}$ and R. Sahai ${ }^{3}$ \\ Received 2004 May 12; accepted 2004 July 29
}

\begin{abstract}
We present deep optical long-slit spectra of the peculiar protoplanetary nebula (PPN) OH 231.8+4.2 obtained with the $6.5 \mathrm{~m}$ Baade Telescope at Las Campanas Observatory (Chile). In addition to the molecular absorption bands characteristic of the M9-10 III star inside OH 231.8+4.2 (QX Pup), we identify lines of the Balmer series in absorption, which do not form in the cool atmospheres of late M-type stars. We also confirm the presence of a blue continuum excess with an intensity that is a factor $\sim 30$ larger than that expected for an M9-10 III star. Our results indicate the presence of a source hotter than QX Pup illuminating $\mathrm{OH} 231.8+4.2$ that is likely a mainsequence star with spectral type A. We discuss how the formation and nebular evolution of $\mathrm{OH} 231.8+4.2$ could have been affected by the presence of a binary system in its core.
\end{abstract}

Subject headings: binaries: general — circumstellar matter — stars: AGB and post-AGB stars: individual $(\mathrm{OH} 231.8+4.2)$ — stars: mass loss — stars: variables: other

\section{INTRODUCTION}

Since its discovery as an $\mathrm{OH}$ maser and infrared source three decades ago (Turner 1971; Wynn-Williams et al. 1974), $\mathrm{OH} 231.8+4.2$ (hereafter $\mathrm{OH} 231.8$ ) has been extensively studied by many authors. OH 231.8 is composed of a bipolar nebula and at least one evolved star. The optical nebula exhibits two broad, line emission lobes in the shape of a calabash (e.g., Bujarrabal et al. 2002). The gas in the lobes has been shockexcited and accelerated up to velocities $\gtrsim 400 \mathrm{~km} \mathrm{~s}^{-1}$ (e.g., Reipurth 1987; Sánchez Contreras et al. 2000a). Inside the broad optical lobes there are two narrow nebulosities seen in dust-scattered starlight and molecular line emission (Sánchez Contreras et al. 2000b; Alcolea et al. 2001; Meakin et al. 2003). One-third of the total nebular mass of OH $231.8\left(M_{\text {total }} \sim 1 M_{\odot}\right)$ is associated with the narrow lobes and is expanding at high velocity. The total scalar momentum of this axial outflow is much higher, by a factor of 1000 , than that which can result from radiation pressure (Sánchez Contreras et al. 1997; Alcolea et al. 2001). The rest of the molecular material is concentrated close to the nebula center and is expanding at low velocity $(\sim 10 \mathrm{~km}$ $\mathrm{s}^{-1}$ ) in the direction perpendicular to the lobes. The association between the nebula and the central evolved star (QX Pup), which is a Mira variable with a spectral type about M9 III (Kastner et al. 1992; Cohen 1981), suggests that the former is the result of the stellar mass loss. It is widely believed that $\mathrm{OH} 231.8$ is a protoplanetary nebula (PPN), i.e., a precursor of a planetary nebula $(\mathrm{PN})$.

The presence of a binary system in the core of $\mathrm{OH} 231.8$ has been postulated by several authors in an attempt to explain, among other peculiarities, the apparently contradictory evolutionary status of QX Pup, which is an asymptotic giant branch (AGB) star, and the surrounding nebula, which shows clear signs of post-AGB evolution such as bipolarity, fast outflows,

\footnotetext{
${ }^{1}$ Division of Physics, Mathematics, and Astronomy, California Institute of Technology, MS 105-24, 1200 East California Boulevard, Pasadena, CA 91125; sanchez@astro.caltech.edu.

2 The Observatories, Carnegie Institution of Washington, 813 Santa Barbara Street, Pasadena, CA 91101; agpaz@ociw.edu.

3 Jet Propulsion Laboratory, California Institute of Technology, MS 183900, 4800 Oak Grove Drive, Pasadena, CA 91109; sahai@eclipse.jpl.nasa.gov.
}

and shocks (see references above). The light from the central source inside $\mathrm{OH} 231.8$, as for most PPNs, is highly obscured along the line of sight by a thick equatorial waist of dust. Therefore, most of our poor knowledge on the central source is indirectly obtained. A blue excess in the scattered continuum suggests the presence of a warm companion (Cohen et al. 1985). In addition, Gómez \& Rodríguez (2001) attribute the misalignment of the $\mathrm{OH}$ and $\mathrm{SiO}$ maser emission distribution to the presence of a binary system. Here, we report the discovery of Balmer absorption lines in the scattered spectrum of $\mathrm{OH} 231.8$, providing a robust observational indication of the presence of at least two stars illuminating the nebula.

\section{OBSERVATIONS AND RESULTS}

Optical long-slit spectra of OH 231.8 were obtained on 2002 March 5 and 6 using the low-dispersion survey spectrograph (LDSS-2) of the $6.5 \mathrm{~m}$ Baade telescope at Las Campanas Observatory (Chile). The detector was a SITe CCD with $2048 \times$ 2048 squared pixels of $24 \mu \mathrm{m}$. The pixel angular scale was 0 " 38 pixel $^{-1}$. The total wavelength coverage is $\sim 3300-6800 \AA$, and the reciprocal dispersion is $1.86 \AA \mathrm{pixel}^{-1}$. We used a 1 ". $4 \times 400^{\prime \prime}$ slit oriented along the symmetry axis of the nebula (at P.A. $=20^{\circ}$; Fig. 1). The total integration time was $3900 \mathrm{~s}$. The data were reduced following standard procedures within IRAF. $^{4}$ We used CuAr lamps to perform wavelength calibration. The spectral resolution achieved (FWHM of the lamp lines) is 7-8 $\AA$. Three photometric standards, LTT2415, LTT4816, and LTT4364, were used for flux calibration. The seeing was $\sim 1^{\prime \prime}$.

The spectrum of $\mathrm{OH} 231.8$ (Figs. 1 and 2) consists of scattered continuum emission, which arises in the narrow reflection lobes, and a series of emission lines, which originate in the broad shock-excited lobes. At red wavelengths, the continuum spectrum shows deep molecular bands (mostly from TiO and VO) characteristic of the late-M Mira star inside $\mathrm{OH} 231.8$, QX Pup ( $(1)$. By comparing the red continuum observed in

\footnotetext{
${ }^{4}$ IRAF is distributed by the National Optical Astronomy Observatories, which are operated by the Association of Universities for Research in Astronomy, Inc., under cooperative agreement with the National Science Foundation.
} 

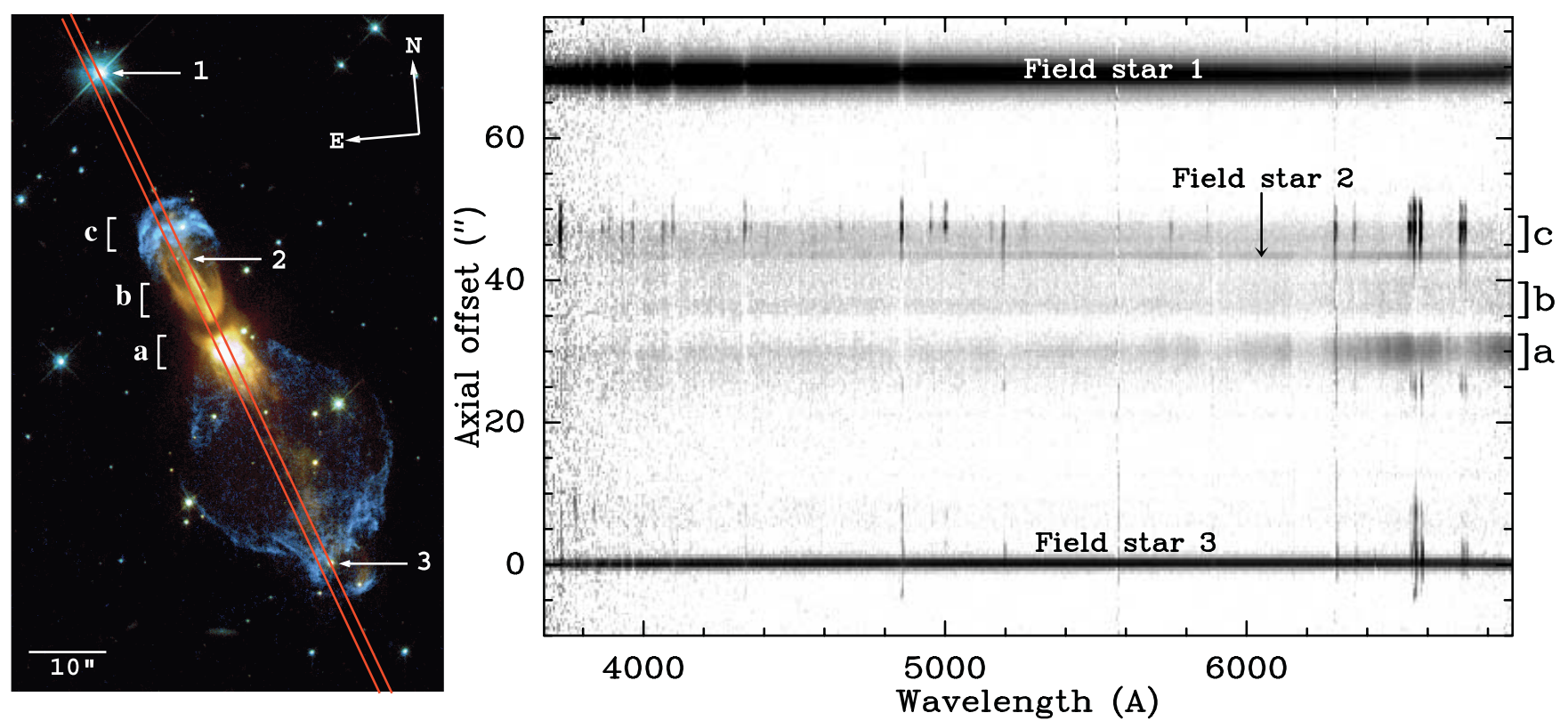

FIG. 1.-Left: False-color image of $\mathrm{OH} 231.8$ in the light of $\mathrm{H} \alpha$ (blue) and $R$ band (yellow) obtained with the Hubble Space Telescope. (This image was originally

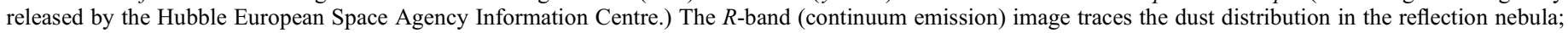

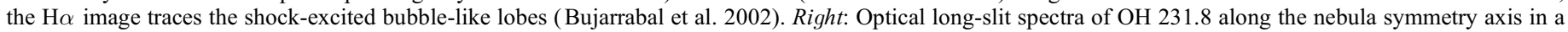
logarithmic flux scale (the slit used in these observations is overlaid on the HST image).

$\mathrm{OH} 231.8$ with spectra of late-type giants in the literature (Jacoby et al. 1984), we derive a spectral type for QX Pup of M10 III (see $\S 3.1$ ). This spectral type is slightly different from (but comparable to) that obtained by Cohen (1981), M9 III, most likely because of small temperature changes resulting from the stellar variability. The continuum flux in the blue considerably exceeds the expected emission from QX Pup at these short wavelengths (see $\S 3.1$ and Fig. 3). In the same spectral region, several Balmer absorption lines are seen. These lines are not expected for an M9-10-type $\operatorname{star}\left(T_{\text {eff }} \sim 2500 \mathrm{~K}\right)$.

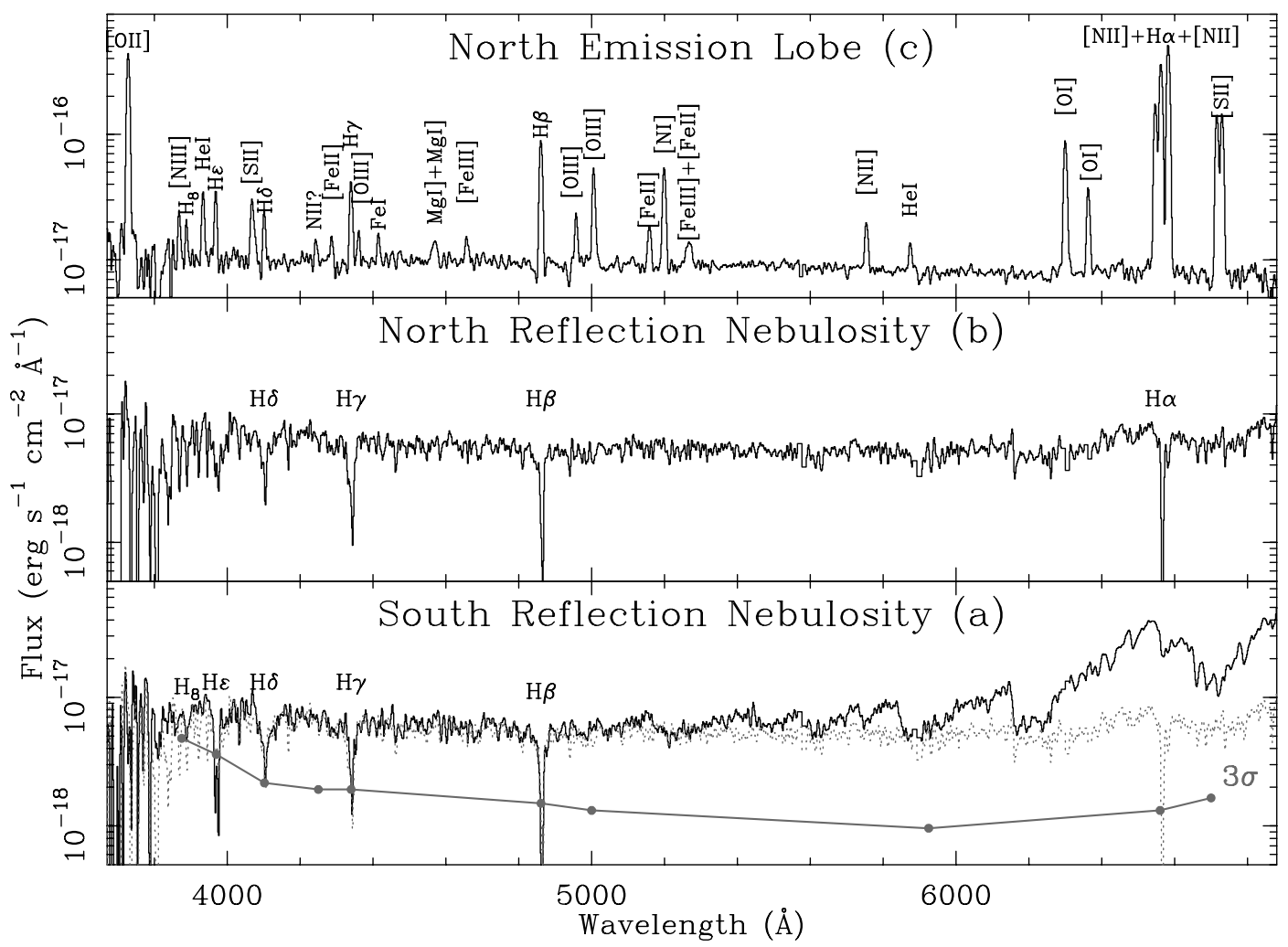

FIG. 2.- Spectra integrated in a region of 4".2 along the slit at positions (a), (b), and (c) (see Fig. 1). In the bottom panel the spectrum in region (b) is plotted again (dotted line) for comparison. The $3 \sigma$ level above the sky is also represented. Note the logarithmic scale in the flux axis. 


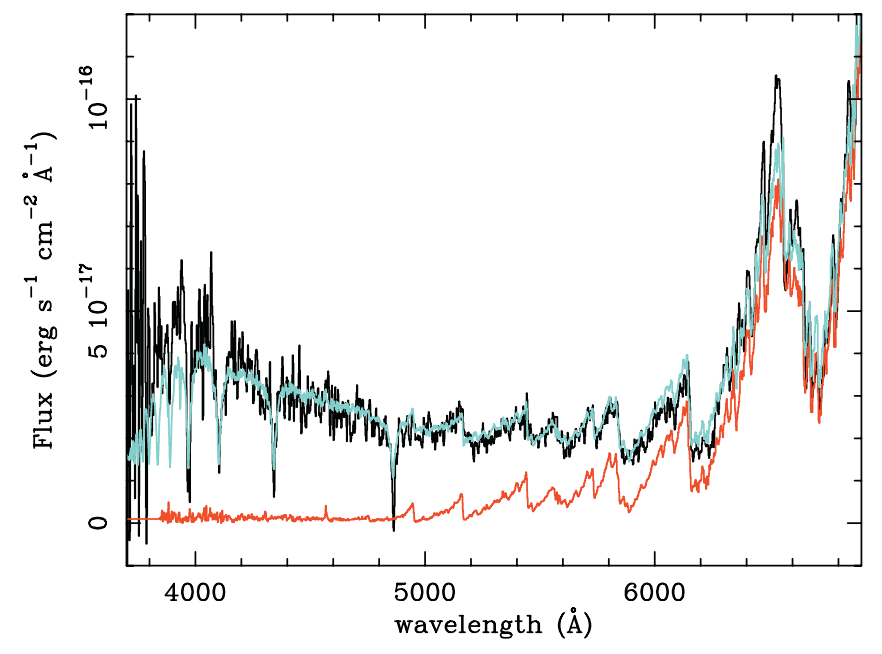

FIG. 3.-Integrated dereddened spectra in the south reflection nebulosity, i.e., region (a) (black). We also show the spectrum of an M10 III star (red) from Fluks et al. (1994) and a composite M10 III+A0 V spectrum (cyan).

The equivalent widths (EWs) of the observed Balmer lines are larger than $10 \AA$ (only a lower limit to EW can be given, since the bottom of the lines is below the $3 \sigma$ level above the sky; Fig. 2). The blue continuum excess and the absorption Balmer lines both indicate that there is a source warmer than QX Pup illuminating the nebula. The Balmer absorption lines are redshifted with respect to the systemic velocity of $\mathrm{OH} 231.8$ in both lobes (e.g., the $\mathrm{H} \beta$ lines are centered at $V_{\text {lsr }}-V_{\text {sys }}>110 \pm$ $80 \mathrm{~km} \mathrm{~s}^{-1}$ ), which is consistent with their scattered nature.

We have identified 27 different emission lines (many of them previously undetected) arising in the north shock-excited emission lobe of $\mathrm{OH} 231.8$. Only the brightest lines are detected in the fainter south emission lobe. From the $\mathrm{H} \alpha / \mathrm{H} \beta$ intensity ratio we derive an extinction $A_{V}=0.7-0.8 \mathrm{mag}$ in the north emission lobe and $A_{V}$ ranging from $\sim 0.6$ to $0.2 \mathrm{mag}$ in the south (the lowest extinction corresponds to the lobe tip) assuming an intrinsic Balmer decrement of $\sim 3$ (Sánchez Contreras et al. 2000a). The emission-line spectrum observed in $\mathrm{OH} 231.8$ is useful for characterizing the shocked gas in the lobes. For example, from the $[\mathrm{N}$ II $] I(6583+6548) / I(5755)$ intensity ratio, $\sim 54.5$, we derive an electron temperature in the north emission lobe $T_{e} \approx 13,000 \mathrm{~K}$ (e.g., Gurzadyan 1997), using an average value of the electron density of $\sim 500 \mathrm{~cm}^{-3}$ (Sánchez Contreras et al. 2000a). The observed continuum in the north emission lobe, region (c), is 1 order of magnitude brighter than the expected nebular continuum, indicating that the former is mostly scattered light. The nebular continuum, which accounts for two-photon, $\mathrm{H}$ I, and He II continuum emission, has been estimated using the ionization parameters by Ferland (1980). Further discussion and analysis of the full emission-line spectrum of $\mathrm{OH} 231.8$ and its shocked lobes is beyond the scope of this paper, which is concerned with the discovery and characterization of the companion of QX Pup and its relevance to the nebular evolution of this peculiar object.

\section{ANALYSIS}

\subsection{The Companion of $Q X$ Pup}

The presence of Balmer absorption lines with EW $>10 \AA$ in the scattered spectrum of $\mathrm{OH} 231.8$ indicates a spectral type A for the companion of QX Pup; B and F stars show Balmer lines with $\mathrm{EW}<10 \AA$ for any luminosity class (e.g.,
Jacoby et al. 1984). Our upper limit on EW of the Ca II K $3933 \AA$ line is $4.5 \AA$, ruling out a spectral type later than A9, for which EW $>4.5 \AA$. The slope of the blue scattered continuum, as estimated from the dereddened spectrum for the south reflection nebulosity (hereafter south $\mathrm{RN}$ ), is most consistent with a spectral type near A0 ( $T_{\text {eff }} \sim 10,000 \mathrm{~K}$; Fig. 3$)$. The extinction used for dereddening is $A_{V}=1.3 \mathrm{mag}$, as derived from the $\mathrm{H}_{2}$ column density toward the south RN $\left(1.3 \times 10^{21} \mathrm{~cm}^{-2}\right.$; Alcolea et al. 2001) and adopting a standard column density to visual extinction ratio of $N_{\mathrm{H}_{2}} / A_{V} \approx 10^{21} \mathrm{~cm}^{-2} \mathrm{mag}^{-1}$ (Huggins \& Glassgold 1982). Our estimate of $A_{V}$ does not include radial extinction, i.e., extinction from the source to the region in which the light is scattered, and, therefore, the total extinction can be larger than 1.3 mag. However, the total extinction has to be $A_{V} \lesssim 2 \mathrm{mag}$, because otherwise the corresponding dereddened spectrum would indicate a spectral type B or earlier, which we have already ruled out based on the strength of the Balmer absorption lines. Although an A0 spectral type seems most consistent with the slope of the continuum, we cannot rule out a cooler A spectral type, since the extinction and scattering dependence with the wavelength in $\mathrm{OH} 231.8$ are not well known.

Assuming that the light from both QX Pup and its warm companion is extinguished and scattered by dust in the same manner and amount, we derive a luminosity for the latter of $\sim 5-30 L_{\odot}$ (for a typical luminosity of the Mira of $\sim 10^{4} L_{\odot}$ ). The relative luminosity of the companion to that of QX Pup has been estimated by fitting the observed scattered spectrum by a composite M10 III+A0 V spectrum (Fig. 3). The range for the luminosity ratio given above results from the fitting of the spectra to the south and north RNs, which have slightly different colors, most likely because of extinction and star variability effects ( $\S 3.2)$. This result suggests that the companion to QX Pup is a main-sequence star. The mass of an A0 V star is $1.5-2.5 M_{\odot}$, and therefore it is consistent with being less evolved than QX Pup, since the latter had a mass in the main sequence of $\sim 3 M_{\odot}$ (Jura \& Morris 1985) and is already in the latest stages of the AGB. (The mass on the main sequence of a B8 star is $>3 M_{\odot}$, and larger for earlier spectral types, which also supports an A spectral type for the companion.) The mass of QX Pup is derived from the turnoff mass of the open cluster NGC 2437 (M 46), to which OH 231.8 most likely belongs. The cluster membership of $\mathrm{OH} 231.8$ is almost certain, because the distance to and radial velocity of $\mathrm{OH} 231.8$ and the cluster are very similar: $d \sim 1300-1500 \mathrm{pc}$ and $V_{\mathrm{lsr}} \sim 30 \mathrm{~km} \mathrm{~s}^{-1}$, respectively, for both systems (see Jura \& Morris 1985, and references therein). The hot companion is not a (first-ascent) giant branch star (although its derived luminosity and temperature are consistent with such a classification), since an A giant star, which has a main-sequence mass of $\sim 4 M_{\odot}$, would now be more evolved than QX Pup.

The star responsible for the blue continuum in $\mathrm{OH} 231.8$ is unlikely to be a field star. The distribution of the blue continuum and Balmer lines along the reflection lobes suggests that the blue star is either (1) inside the equatorial waist of dust and, therefore, is a companion of QX Pup, or (2) outside and behind the dusty equatorial disk. The latter case is, however, unlikely, because the star would have to lie exactly in a small region behind the disk and also because its luminosity would have to be a factor $\gtrsim 800$ larger than that derived above. The larger luminosity, which would imply the star is a post-mainsequence star, is needed to account for the larger distance of a star outside the reflection lobes (considering that the typical separation between stars in an open cluster is $2-3$ lt-yr, i.e., 

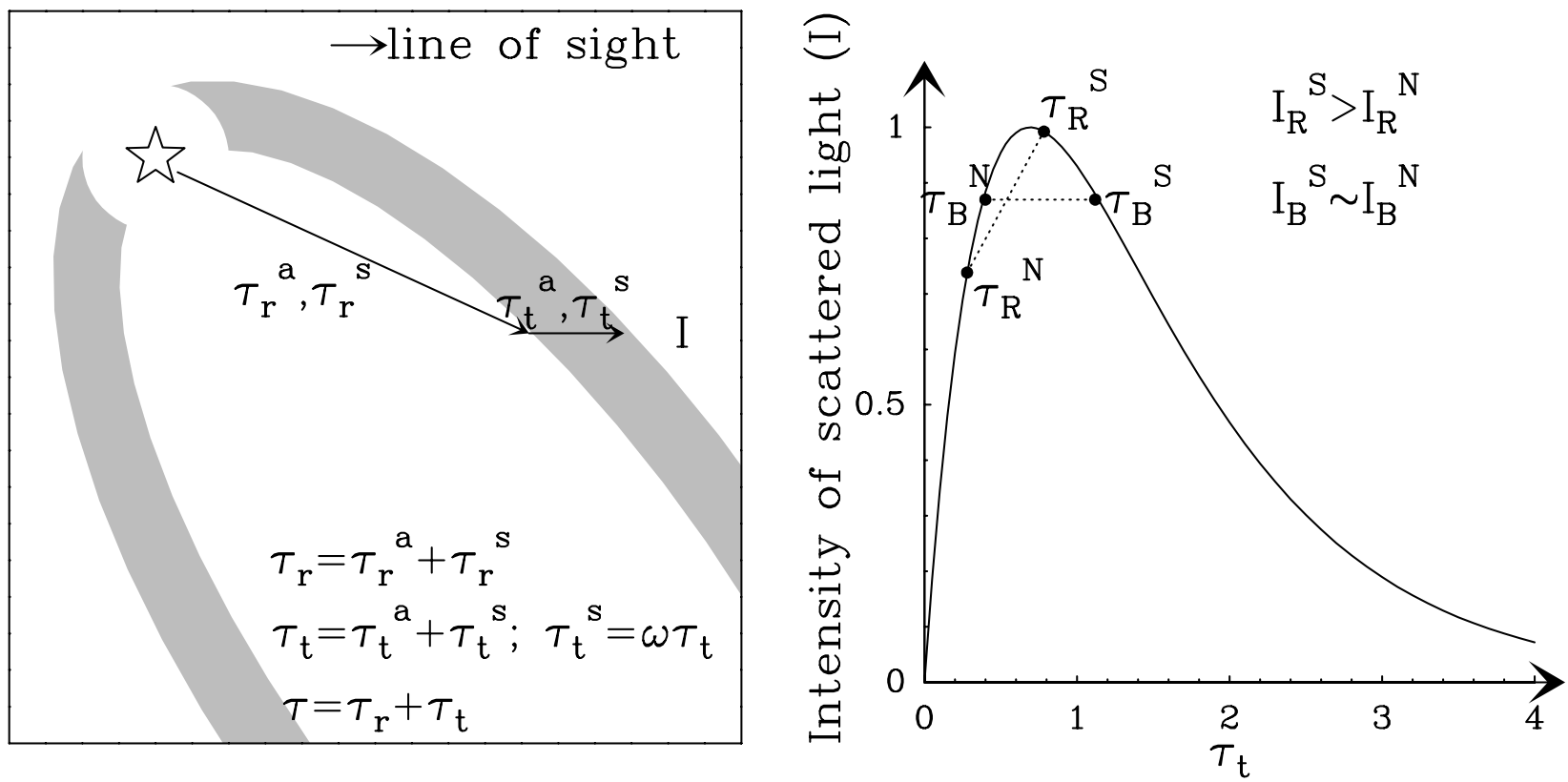

FIG. 4.-Left: Cartoon of one of the lobes of $\mathrm{OH} 231.8$ showing the light path from the central source (represented by the star) to and through the region in which the light is predominantly scattered. The extinction optical depths, including absorption (superscript $a$ ) plus scattering (superscript $s$ ), along the radial and tangential directions are denoted by $\tau_{r}$ and $\tau_{t}$, respectively. The albedo is denoted by $\omega$. Right: Relative intensity of the scattered light $(I)$ as a function of $\tau_{t}$. We show a possible situation in which $\tau_{t}$ is larger for the south reflection nebulae than for the north (superscripts $S$ and $N$, respectively) that would yield a similar intensity of the scattered light in the blue (subscript $B$ ) for the two lobes but a larger intensity in the red (subscript $R$ ) for the south $(\S 3.2)$.

$\sim 100^{\prime \prime}$ at a distance of $1500 \mathrm{pc}$ ) and a larger extinction of its light, which would have to travel across both the front and back lobe walls as well as the dusty halo around the central disk (Alcolea et al. 2001). The close presence of an AGB star (QX Pup) and a post-main-sequence field star is less likely than a binary system formed by an AGB and a main-sequence star.

\subsection{The Color of the South and North Reflection Lobes}

The south $\mathrm{RN}$ is redder than the north $\mathrm{RN}$; e.g., the $6500 / 4500 \AA$ intensity ratio in the former is $\sim 6$ compared with $\sim 1.3$ in the latter (Fig. 2). In addition, the red continuum in the south $\mathrm{RN}$ is brighter than in the north by a factor of $\sim 8$ (i.e., $\Delta m_{R} \sim 2.3 \mathrm{mag}$, after subtracting the contribution of the companion to the continuum flux), whereas the blue continuum flux is similar in both RNs. This could result from a combined effect of (1) the variability of the central Mira and the different lighttravel time from the north and south RNs to the observer, and (2) larger extinction optical depths through the lobe walls in the south than in the north RN.

(1) Adopting a nebular inclination $i=36^{\circ}$ (Shure et al. 1995), the light-travel difference between the south and north $\mathrm{RN}$ of $\mathrm{OH} 231.8$ is $\sim 50$ days, which represents a phase of 0.07 of the QX Pup pulsational period ( $P \sim 700$ days; Kastner et al. 1992). In this time interval, the optical emission of a long-period Mira star can vary up to $\sim 3$ mag (e.g., Mattei 1997, and references therein), which is sufficient (but see next paragraph) to explain the $\Delta m_{R} \sim 2.3 \mathrm{mag}$ difference between the south and north RN. Since the south RN is farther away from us than the north $\mathrm{RN}$, the scattered continuum in the former was emitted at an earlier epoch, and, therefore, the fainter red continuum in the north is consistent with QX Pup being in a phase of declining optical brightness.

Nevertheless, if the color difference is due only to the variability of QX Pup, then at some epochs we should expect the south lobe to be fainter in the red than the north. The south lobe is, however, always the brightest in the $R$ band in the few optical data published so far (Cohen et al. 1985; Reipurth 1987; Sánchez Contreras et al. 2000a). In the $K$ band, which is also dominated by scattered light, and which has been monitored over a 3 yr period (Kastner et al. 1992), the south lobe is always brighter than the north by $0.3-1 \mathrm{mag}$, depending on the phase. This suggests that there are some intrinsic differences in the mass of dust in the north and south lobes.

(2) The intensity of the scattered continuum in the reflection lobes, $I$, is given by

$$
I \propto I_{0} e^{-\tau_{r}} e^{-\tau_{t}}\left(1-e^{-\tau_{t}^{s}}\right) \propto e^{-\tau_{t}}\left(1-e^{-\omega \tau_{t}}\right),
$$

where $I_{0}$ is the emission from the central source, $\tau_{r}$ and $\tau_{t}$ are, respectively, the extinction (absorption plus scattering) optical depth from the central source to the scattering region (radial) and from the scattering region to the observer (tangential), $\tau_{t}^{s}$ is the tangential scattering optical depth at the observed region, and $\omega$ is the grain albedo (Fig. 4). This expression is valid for scattering occurring predominantly in the lobe walls, which is a reasonable approximation for $\mathrm{OH} 231.8$ (Bujarrabal et al. 2002; Kastner \& Weintraub 1995). The relatively low extinction across the lobe walls (along the line of sight) in regions (a) and (b) $\left(\tau_{t} \sim A_{V}=1.3 \mathrm{mag} ; \S 3.1\right)$ implies $\tau_{t}^{s}<1$, since the absorption and scattering optical depths are expected to be of the same order. According to equation (1), I has an absolute maximum around $\tau_{t}^{\max } \sim 0.8$, and, therefore, two different values of $\tau_{t}$ (one at each side of the maximum) reproduce the same intensity of the scattered continuum. This could explain why the blue continuum brightness is similar for the north and south $\mathrm{RN}$ even if $\tau_{t}^{s}$ is different through each lobe. In addition, if $\tau_{t}^{s}$ is larger for the south RN than for the north, then the former would be the brightest in the red, as observed, since the optical depth decreases with wavelength.

Finally, we cannot rule out the fainter red continuum in the north $\mathrm{RN}$ resulting from a larger radial extinction of the light 
of QX Pup (but not of the companion) in the north polar direction than in the south.

\section{NEBULAR FORMATION AND EVOLUTION}

The interaction between fast, collimated (jetlike) winds and the slow AGB envelope is believed to be the most likely mechanism for the formation and evolution of the bipolar nebula in $\mathrm{OH} 231.8$ (Bujarrabal et al. 2002, and references therein) in particular, and most bipolar planetary nebulae in general (Sahai \& Trauger 1998; see also Balick \& Frank 2002 for a recent review). Our detection of a companion to QX Pup can now help us to fully understand the formation of this unique object, since binarity is a widely accepted mechanism for producing fast collimated winds in dying stars (Morris 1987; Soker \& Rappaport 2000, and references therein). We propose that most of the circumstellar material in $\mathrm{OH} 231.8$ is the result of mass loss from the central Mira, QX Pup, whose slow, but dense, AGB wind is partially trapped by its compact companion in the form of an accretion disk, and re-ejected by the latter as a collimated, fast $\left(\gtrsim 400 \mathrm{~km} \mathrm{~s}^{-1}\right)$ wind in the direction perpendicular to the orbital plane. The hydrodynamic interaction of both winds results in a series of shocks that shape the bipolar lobes and accelerate the lobe material to velocities of up to $\sim 400 \mathrm{~km} \mathrm{~s}^{-1}$ with respect to the central source. This scenario is supported by the following facts.

The average mass-loss rate that gave rise to the massive $\left(\sim 1 M_{\odot}\right)$ molecular envelope in $\mathrm{OH} 231.8$ is estimated to be $10^{-4} M_{\odot} \mathrm{yr}^{-1}$ (Alcolea et al. 2001). Such a high mass-loss rate is only expected in the latest stages of the AGB phase, and, therefore, QX Pup is more likely to be responsible for the ejection of most of the material in $\mathrm{OH} 231.8$ than its companion. A-type main-sequence stars (in the so-called instability strip of the HR diagram) do lose mass through pulsationally driven winds; however, the mass-loss rates observed are $\lesssim 10^{-8} M_{\odot}$ $\mathrm{yr}^{-1}$ (e.g., Lanz \& Catala 1992, and references therein).

As an AGB star, QX Pup is unlikely to blow the fast jetlike wind. First, AGB winds are spherical or, at most, show very small departures from such symmetry (Neri et al. 1998), and second, stellar winds have ejection velocities generally of the same order as the escape velocity, which for QX Pup is $\sim 7-$ $10 \mathrm{~km} \mathrm{~s}^{-1}$ (Sánchez Contreras et al. 2000b, 2002). Since the escape velocities for main-sequence stars are much higher (several $\times 10^{2} \mathrm{~km} \mathrm{~s}^{-1}$ ), the companion of QX Pup is more likely to have blown the fast wind in $\mathrm{OH} 231.8$.

This fast wind is thought to have transferred a large amount of linear momentum $\left(\sim 10 \mathrm{M}_{\odot} \mathrm{km} \mathrm{s}^{-1}\right)$ to the bipolar lobes in only $\sim 100 \mathrm{yr}$ approximately $800 \mathrm{yr}$ ago (Alcolea et al. 2001). For an expansion velocity of the fast wind of $\sim 500-1000 \mathrm{~km}$ $\mathrm{s}^{-1}$, the above figures imply a mass-loss rate of $\dot{M} \sim 10^{-4} M_{\odot}$ $\mathrm{yr}^{-1}$, which is again much larger than the typical values of $\dot{M}$ for mass-losing AV stars. Therefore, the fast wind must result from the accretion of the dense wind of QX Pup into an accretion disk around the companion.

We have estimated a mass accretion rate from the AGB wind to the accretion disk in $\mathrm{OH} 231.8$ of $\dot{M}_{\mathrm{AGB} \rightarrow \text { disk }}=6.7 \times$ $10^{-6} M_{\odot} \mathrm{yr}^{-1}$, using equation (3) of Morris (1990) and assuming an AGB wind outflow speed of $10 \mathrm{~km} \mathrm{~s}^{-1}$, a mass of the primary (companion) $M_{1}=1 M_{\odot}\left(M_{2}=2 M_{\odot}\right)$, and an orbital separation of $r=50 \mathrm{AU}$. If the A star is at the large separation ( $\sim 1000 \mathrm{AU})$ inferred from the relative position of the Mira and the center of the $\mathrm{OH}$ maser outflow (Gómez \& Rodríguez 2001), it is too distant from the primary to produce a bipolar outflow in any model, and we do not consider this possibility further. We have assumed $r=50$ AU because the presence of $\mathrm{SiO}, \mathrm{OH}$, and $\mathrm{H}_{2} \mathrm{O}$ maser emission in $\mathrm{OH} 231.8$ imposes a lower limit to the orbital separation of about 50 AU (Schwarz et al. 1995). However, the above number is a very rough estimate based on a statistical consideration of the selective presence/absence of $\mathrm{SiO}, \mathrm{H}_{2} \mathrm{O}$, and $\mathrm{OH}$ masers in a sample of symbiotic stars; Schwarz et al. further argue that tidal disruption of maser sites is the most important mechanism for close (i.e., separation $\lesssim 10 \mathrm{AU}$ ) binaries, hence the orbital separation in $\mathrm{OH} 231.8$ is very likely at least $10 \mathrm{AU}$. Since only $1 \%-10 \%$ of the accreted mass is usually ejected in the fast wind, we require a much higher mass accretion rate from the disk to the compact A V star in $\mathrm{OH} 231.8$, of about $\dot{M}_{\text {disk } \rightarrow \text { star }} \approx 10^{-3} M_{\odot}$ $\mathrm{yr}^{-1}$, given the $\sim 10^{-4} M_{\odot} \mathrm{yr}^{-1}$ mass-loss rate of its fast wind. In order to explain such a high value of $\dot{M}_{\text {disk } \rightarrow \text { star }}$, we propose that OH 231.8 went through an "FU Ori-type" outburst, as has been observed in many young stellar objects (e.g., Hartmann 1998). These outbursts are short periods of increased accretion rates (by several orders of magnitude), where $\dot{M}_{\text {disk } \rightarrow \text { star }}$ reaches values up to $\sim 10^{-4} M_{\odot} \mathrm{yr}^{-1}$, and are thought to be due to an increase of the viscosity in the disk that causes the disk to empty itself onto the companion in a short timescale $(\sim 100 \mathrm{yr})$. Before (and after) the outburst, matter piles up in the disk steadily at a relatively low rate until a rapid and massive accretion event occurs, resulting in the kind of massive fast wind seen in $\mathrm{OH} 231.8$. At present, $\mathrm{OH} 231.8$ is most likely in a period of steady accretion at a low rate, given the lack of any strong signature of accretion (e.g., $\mathrm{H} \alpha$ emission). This is also in agreement with the fact that the fast wind and the consequent nebular acceleration seem to have already ended (see Alcolea et al. 2001, and above).

An alternative mass-loss outburst phenomenon due to novalike explosions, resulting from the accretion of disk matter onto the surface of a white dwarf companion, has been proposed earlier to explain the complex light curves and axial outflows in symbiotic stars (Soker \& Rappaport 2000; Corradi et al. 2000, and references therein). Such outbursts, which can last up to $100 \mathrm{yr}$, can also result in high-velocity ejections that can entrain nebular material and accelerate it to several $\times 100 \mathrm{~km} \mathrm{~s}^{-1}$.

The work described in this paper has added an exciting new dimension to the investigation of key objects in the study of mass-losing AGB stars, by using very deep spectroscopic observations to detect and characterize the spectrum at short wavelengths, where the light from the cool primary AGB star is minimized. We now need to repeat such observations, at several epochs that sample the light cycle of $\mathrm{OH} 231.8$, in order to determine how the relative color and magnitude of the south and north lobes change with phase, and with higher sensitivity and better spectral resolution, so as to be able to measure accurately the strengths of various diagnostic lines. We may, for example, find changes in the blue spectrum that require a significant luminosity contribution from the inferred accretion disk. Although we have presented the simplest model that explains our observational results, so little is directly known about the region around the stellar vicinity in objects like $\mathrm{OH} 231.8$ that further detailed observations will either provide important additional support for our model or force the generation of new and unconventional ones.

Finally, our discovery of a companion to QX Pup in $\mathrm{OH}$ 231.8 constitutes the most direct detection of a binary system among the relatively sparse list of post-AGB objects that are suspected to host multiple stellar systems (e.g., Bond 2000; Van Winckel 2001; de Marco et al. 2004, and references therein). We have proposed a possible scenario in which mass accretion onto the companion plays an important role in the 
ejection of $\mathrm{OH} 231.8$ 's bipolar outflows. However, our findings do not allow us to generalize about the role of binarity in the shaping of post-AGB objects, because it is not yet clear whether $\mathrm{OH} 231.8$ is a unique object (see Alcolea et al. 2001 for a summary of $\mathrm{OH} 231.8$ 's properties that are not common in PPNs) or an extreme example of a more general phenomenon (e.g., in a large population of binaries produced by the star formation processes that make low and intermediate-mass stars, binaries such as $\mathrm{OH} 231.8$, with a total main-sequence mass of 4.5-5.5 $M_{\odot}$, may be relatively rare).
We are very grateful to B. F. Madore for kindly providing part of his telescope time to this project. The anonymous referee is also thanked for valuable suggestions. This work was performed at California Institute of Technology. C. S. C. and A. G. d. P. are respectively financed by NSF Grant 9981546 to Owens Valley Radio Observatory and NASA Galaxy Evolution Explorer. R. S. is partially financed for this work by NASA Long Term Space Astrophysics grant 399-2061-00-00 and by the Space Telescope Science Institute through proposals GO-08326.01-A and GO-09101.01-A.

\section{REFERENCES}

Alcolea, J., Bujarrabal, V., Sánchez Contreras, C., Neri, R., \& Zweigle, J. 2001, A\&A, 373, 932

Balick, B., \& Frank, A. 2002, ARA\&A, 40, 439

Bond, H. E. 2000, in ASP Conf. Ser. 199, Asymmetrical Planetary Nebulae II: From Origins to Microstructures, ed. J. H. Kastner, N. Soker, \& S. Rappaport (San Francisco: ASP), 115

Bujarrabal, V., Alcolea, J., Sánchez Contreras, C., \& Sahai, R. 2002, A\&A, 389,271

Cohen, M. 1981, PASP, 93, 288

Cohen, M., Dopita, M. A., Schwartz, R. D., \& Tielens, A. G. G. M. 1985, ApJ, 297, 702

Corradi, R. L. M., Livio, M., Schwarz, H. E., \& Munari, U. 2000, in ASP Conf. Ser. 199, Asymmetrical Planetary Nebulae II: From Origins to Microstructures, ed. J. H. Kastner, N. Soker, \& S. Rappaport (San Francisco: ASP), 175

de Marco, O., Bond, H. E., Harmer, D., \& Fleming, A. J. 2004, ApJ, 602, L93

Ferland, G. J. 1980, PASP, 92, 596

Fluks, M. A., Plez, B., The, P. S., de Winter, D., Westerlund, B. E., \& Steenman, H. C. 1994, A\&AS, 105, 311

Gómez, Y., \& Rodríguez, L. F. 2001, ApJ, 557, L109

Gurzadyan, G. A. 1997, The Physics and Dynamics of Planetary Nebulae (Berlin: Springer)

Hartmann, L. 1998, Accretion Processes in Star Formation (Cambridge: Cambridge Univ. Press)

Huggins, P. J., \& Glassgold, A. E. 1982, ApJ, 252, 201

Jacoby, G. H., Hunter, D. A., \& Christian, C. A. 1984, ApJS, 56, 257

Jura, M., \& Morris, M. 1985, ApJ, 292, 487

Kastner, J. H., \& Weintraub, D. A. 1995, AJ, 109, 1211

Kastner, J. H., Weintraub, D. A., Zuckerman, B., Becklin, E. E., McLean, I., \& Gatley, I. 1992, ApJ, 398, 552

Lanz, T., \& Catala, C. 1992, A\&A, 257, 663

Mattei, J. A. 1997, J. AAVSO, 25, 57

Meakin, C. A., Bieging, J. H., Latter, W. B., Hora, J. L., \& Tielens, A. G. G. M. 2003, ApJ, 585, 482

Morris, M. 1987, PASP, 99, 1115 1990, in From Miras to Planetary Nebulae: Which Path for Stellar Evolution? (Gif-sur-Yvette: Editions Frontières), 520

Neri, R., Kahane, C., Lucas, R., Bujarrabal, V., \& Loup, C. 1998, A\&AS, 130,1

Reipurth, B. 1987, Nature, 325, 787

Sahai, R., \& Trauger, J. T. 1998, AJ, 116, 1357

Sánchez Contreras, C., Bujarrabal, V., \& Alcolea, J. 1997, A\&A, 327, 689

Sánchez Contreras, C., Bujarrabal, V., Miranda, L. F., \& Fernández-Figueroa, M. J. 2000a, A\&A, 355, 1103

Sánchez Contreras, C., Bujarrabal, V., Neri, R., \& Alcolea, J. 2000b, A\&A, 357,651

Sánchez Contreras, C., Desmurs, J. F., Bujarrabal, V., Alcolea, J., \& Colomer, F. 2002, A\&A, 385, L1

Schwarz, H. E., Nyman, L.-A., Seaquist, E. R., \& Ivison, R. J. 1995, A\&A, 303,833

Shure, M., Sellgren, K., Jones, T. J., \& Klebe, D. 1995, AJ, 109, 721

Soker, N., \& Rappaport, S. 2000, ApJ, 538, 241

Turner, B. E. 1971, Astrophys. Lett., 8, 73

Van Winckel, H. 2001, Ap\&SS, 275, 159

Wynn-Williams, C. G., Becklin, E. E., \& Neugebauer, G. 1974, ApJ, 187, 473 